\title{
Phase synchronization in bidirectionally coupled optothermal devices
}

\author{
R. Herrero \\ Departament de Física i Enginyeria Nuclear, Universitat Politècnica de Catalunya, Comte Urgell 187, 08036 Barcelona, Spain \\ M. Figueras, F. Pi, and G. Orriols \\ Departament de Física, Universitat Autònoma de Barcelona, 08193 Bellaterra, Spain
}

(Received 21 May 2002; published 27 September 2002)

\begin{abstract}
We present the experimental observation of phase synchronization transitions in the bidirectional coupling of chaotic and nonchaotic oscillators. A variety of transitions are characterized and compared to numerical simulations of a time delayed model. The characteristic $2 \pi$ phase jumps usually appear during the transitions, specially in those clearly associated with a saddle-node bifurcation. The study is done with pairs of optothermal oscillators linearly coupled by heat transfer.
\end{abstract}

DOI: 10.1103/PhysRevE.66.036223

PACS number(s): 05.45.Xt, 42.50.Ar

\section{INTRODUCTION}

Synchronization of coupled chaotic oscillators has recently been the object of intensive research and different types of synchronization have been described [1,2]. One of the relevant behaviors expected for weak couplings is the phase synchronization (PS) phenomenon, i.e., the synchronization of phases while amplitudes have not to be necessarily correlated. The transition to PS when the coupling is increased was first observed in mutually coupled Rössler models by Rosenblum et al. [3]. A characteristic feature of the observed transition is the occurrence of intermediate states in which the phase difference of the oscillators remains almost fixed, for finite time intervals suddenly interrupted by $2 \pi$ phase jumps and the mean frequency of such jumps decreases with increasing the coupling towards the PS state $[3,4]$. The transition to PS has been numerically studied with different models considering two or more oscillators and it has been associated with a variety of dynamical bifurcations [4-10]. Phase jumps are almost always observed in the numerically simulated PS transitions but with a variety of scaling properties that seem related to the kind of underlying bifurcation $[3,4,11,9]$ and the influence of noise [12].

Experimental demonstrations of PS to an external periodic pacing have been reported for a variety of systems exhibiting chaotic evolutions [13-16] and irregular biological rhythms $[17,18]$. The PS between unidirectionally coupled chaotic oscillators has been also reported $[19,20]$ and the concept of phase synchrony has been used for the characterization of rather complex oscillatory behaviors such as those observed in brains [21-23]. The first experimental observation of a transition to PS in bidirectionally coupled oscillators has been reported very recently [24]. In this case the transition happens via phase jumps occurring upwards or downwards irregularly, in a similar way as numerically detected in coupled hyperchaotic Rössler oscillators, where PS has been related to type-II inttermitency [9], and with scaling properties agreeing well with those observed in coupled Rössler models [4].

In this work we present a detailed experimental analysis of a number of synchronization transitions observed in pairs of bidirectionally coupled oscillators, including intermediate states with phase jumps and PS states with uncorrelated amplitudes. The experiment is done with a kind of optothermal nonlinear oscillators linearly coupled by heat transfer and we have used pairs of two- and three-dimensional oscillators that exhibit periodic and chaotic evolutions when isolated, respectively. In all of the cases, the coupled elements are nearly similar but not identical, with slight differences in both oscillating frequencies and steady-state solutions. Numerical simulations in reasonable agreement with the experimental results indicate that we have observed PS transitions clearly associated either with a cyclic saddle-node bifurcation or with a secondary Hopf bifurcation. Nevertheless, the analysis points out a rich variety of PS transitions without a clear relation with a specific bifurcational process.

\section{NONLINEAR DEVICE AND EXPERIMENTAL SETUP}

The nonlinear oscillators are based on the so-called optothermal bistability with localized absorption (BOITAL) and they have been described in detail elsewhere [25-27]. A BOITAL device consists of a Fabry-Pérot cavity, where the input mirror is a partially absorbing film, the rear mirror is a high-reflection dielectric coating, and the spacer between mirrors is constituted by $N$ transparent layers with alternatively opposite thermo-optic coefficients. The cavity is illuminated with a focalized laser beam and the reflected power is detected with a photodiode. The light absorption in the input mirror is affected by the interference effects, as described by the Airy function, and it constitutes the nonlinearity of the system. The device presents a multiple stationary solution associated with the periodicity of the nonlinear function. The effective dimension of the device dynamics is $N$ and the system is able to experience up to $N-1$ different Hopf bifurcations due to the competition and time delay between the contributions of the various layers to the light phase shift within the cavity $[25,26]$.

As shown in Fig. 1, different oscillators separated by a certain distance can be created by focusing parallel light beams onto the same transversally extended optical device. The nonlinear elements are coupled by heat propagation through the cavity spacer and the separation distance $d$ may be used to adjust the coupling strength. The oscillators have 


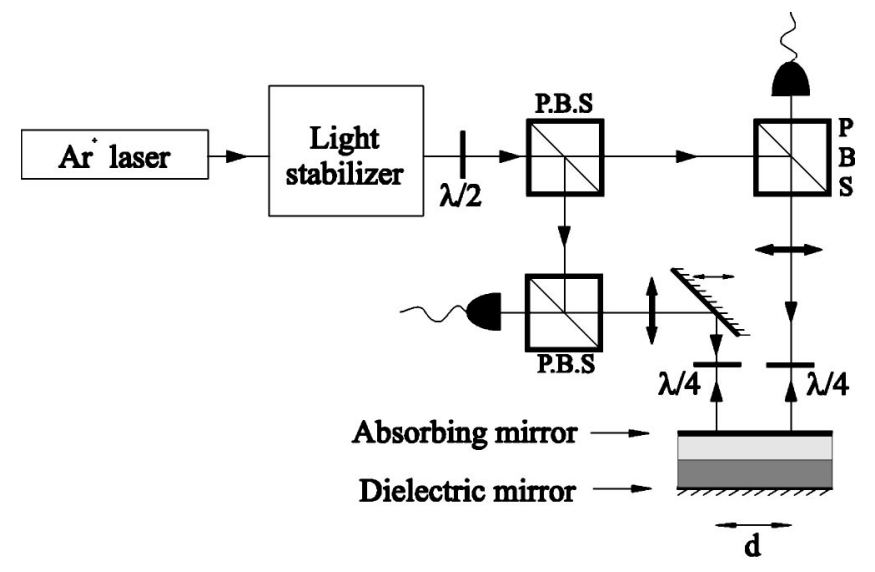

FIG. 1. Experimental setup where two light beams focalized on the BOITAL device create a pair of nonlinear oscillators. The separating distance can be changed with the mobile mirror to modify the thermal coupling strength. The polarizing beam splitters (PBS) and retarder plates are used to regulate the relative powers of the two beams, to optically isolate the laser cavity from the nonlinear device and to obtain independent detection of the reflected light from the two oscillators.

the same cavity parameters, although slight optical path differences may occur due to nonuniform thicknesses and to different light beam inclinations, and significant differences may be introduced with the incident light powers. The light beam of $488 \mathrm{~nm}$ is provided by an argon-ion laser with the power fluctuations reduced to less than $0.1 \%$ by means of an electro-optic modulator subjected to feedback. The light beam is divided in a polarizing beam splitter, the relative powers of the output beams are regulated by changing the input polarization with a $\lambda / 2$ plate and, finally, the two beams are circularly polarized with properly oriented $\lambda / 4$ plates and focalized to a $50-\mu \mathrm{m}$-diameter spot. The light polarization is not relevant for the nonlinear device because it contains isotropic materials only and almost normal incidences are always used. The $\lambda / 4$ plate, jointly with an additional polarizing beam splitter, allow us to collect the reflected light from the nonlinear device on a photodiode and, at the same time, they avoid any return to the laser cavity that could produce instabilities into the laser oscillator. The nonlinear device is placed on a thermoelectric plate to define better the background temperature and to avoid uncontrolled changes in the cavity optical path.

In the case of nearly equal oscillators the transition to PS is expected to be observed from very weak to stronger couplings $[3,4]$. The coupling is considered weak when the limit cycle attraction, given by the system nonlinearity, is much larger than the coupling effects. In this way, transitions expected as we move from weak to strong couplings could also be observed by decreasing the strength of the system nonlinearity. In our case, the nonlinearity strength directly depends on the input light power, which can be easily varied independently of the rest of parameters, and the PS transitions have been observed by decreasing the total incident power for a fixed separation distance.

The phase difference $\theta$ between the two subsystems is simply determined from the relative position of the individual oscillatory maxima and, since it exhibits time variations very much slower than the oscillation periods, this method provides us with equivalent results as those using the Hilbert transform of the signal [3].

\section{TWO COUPLED TWO-DIMENSIONAL SUBSYSTEMS}

Two different routes to phase synchronization have been clearly observed in the case of two coupled two-dimensional subsystems, one transition through phase jumps and another one through a secondary Hopf bifurcation. In the experiment the two transition types have been obtained by changing the layer thicknesses and separation distance between subsystems and they have been also reproduced in numerical simulations of a simple model of the coupled system.

The behavior of a BOITAL cavity is well described by the homogeneous heat equation, subject to a nonlocal and nonlinear boundary condition [25]. This physical model can be reduced to a dimensionless model of order equal to the number of layers and where the variables $\psi_{j}$ are the light phase shifts due to temperature rises in each one of the layers [26]. Every $\psi_{j}$ is proportional to the averaged temperature rise and to the thermo-optical coefficient $\eta_{j}$ of the corresponding layer. A model for a pair of thermally coupled two-layer oscillators has been already used in Ref. [28] to describe the amplitude death effect with very acceptable results. The model is made up by the following four equations:

$$
\begin{aligned}
\frac{d \psi_{1}^{a}}{d t}= & -b_{11} \psi_{1}^{a}-b_{12} \psi_{2}^{a}+G_{1} A\left(\psi^{a}\right) \psi_{e}^{a}+c_{1} \psi_{1}^{b}\left(t-\tau_{1}\right) \\
& -\left(c_{1}+c_{2}\right) \psi_{1}^{a}, \\
\frac{d \psi_{2}^{a}}{d t}= & -b_{21} \psi_{1}^{a}-b_{22} \psi_{2}^{a}+G_{2} A\left(\psi^{a}\right) \psi_{e}^{a}+c_{2} \frac{\eta_{2}}{\eta_{1}} \psi_{1}^{b}\left(t-\tau_{2}\right), \\
\frac{d \psi_{1}^{b}}{d t}= & -b_{11} \psi_{1}^{b}-b_{12} \psi_{2}^{b}+G_{1} A\left(\psi^{b}\right) \psi_{e}^{b}+c_{1} \psi_{1}^{a}\left(t-\tau_{1}\right) \\
& -\left(c_{1}+c_{2}\right) \psi_{1}^{b}, \\
\frac{d \psi_{2}^{b}}{d t}= & -b_{21} \psi_{1}^{b}-b_{22} \psi_{2}^{b}+G_{2} A\left(\psi^{b}\right) \psi_{e}^{b}+c_{2} \frac{\eta_{2}}{\eta_{1}} \psi_{1}^{a}\left(t-\tau_{2}\right),
\end{aligned}
$$

where superindexes $a$ and $b$ denote the two subsystems. $\psi^{x}$ $=\psi_{0}^{x}+\sum_{j=1}^{2} \psi_{j}^{x}$ is the total round-trip phase shift, $\psi_{0}^{x}$ is the light phase shift in the absence of laser heating, and $\psi_{e}^{x}$ is the normalized incident light power for the oscillator $x$. The nonlinear function $A\left(\psi^{x}\right)$ is the Airy function describing the light interference within the absorbing mirror, it depends on the mirror parameters only and is the same for both oscillators except for possible differences in the initial phases $\psi_{0}^{x}$. The coefficients $b_{i j}$ and $G_{i}$ depend on parameters of the spacing layers as indicated in Ref. [26] and are common to both oscillators. The diffusive coupling is simply described by considering the heat flow from the first layer of each oscillator, i.e., the layer next to the absorbing film, towards both layers of the other oscillator. The heat flow from the 
second layer is not considered because this layer has temperature variations much more lower than the first one. The finite speed of heat propagation is taken into account by introducing two different time delays $\tau_{1}=d^{2} / D_{1}$ and $\tau_{2}$ $=\left(d^{2}+g_{1}^{2}\right) / D_{1}$ into the terms describing the heat arrival at the two layers, respectively. The coupling coefficients are taken as $\mathrm{c}_{1}=f\left(K_{1} / d^{2}\right)$ and $\mathrm{c}_{2}=f K_{1} /\left(d^{2}+g_{1}^{2}\right)$, where $D_{1}$, $K_{1}$, and $g_{1}$ are the thermal diffusivity, thermal conductivity and thickness of the first layer, respectively, and $f$ is a constant factor that relates the heat transfer with phase variations.

\section{A. Transition through phase jumps}

We generate a pair of oscillators, $a$ and $b$, in a two-layer device made of glass and sunflower oil, with thicknesses $g_{1,2}=400 \mu \mathrm{m}$ and $120 \mu \mathrm{m}$ and effective thermo-optical coefficients $\eta_{1,2}=10^{-5} \mathrm{~K}^{-1}$ and $-3.4 \times 10^{-4} \mathrm{~K}^{-1}$, respectively. The input mirror of the cavity is a $7-\mathrm{nm}$ nickelchrome film with reflections of about 0.2 and a transmission of about 0.4 and the rear dielectric mirror has a high reflection (>0.98). The incident laser beams with powers $P_{e}^{a}$ and $P_{e}^{b}$ are adjusted to be equal within a $4 \%$ of error and with a separating distance $d$ equal to $6 \mathrm{~mm}$. Maintaining the ratio $P_{e}^{a} / P_{e}^{b} \simeq 1$ and increasing the total input power $P_{e}=P_{e}^{a}$ $+P_{e}^{b}$, the reflected powers, $P_{R}^{a}$ and $P_{R}^{b}$, start to oscillate at $2.33 \mathrm{~Hz}$ through a Hopf bifurcation for $P_{e}=73.1 \mathrm{~mW}$, while without coupling the individual units begins to oscillate for input power values $\left(P_{e}^{a}, P_{e}^{b}\right)$ equal to $(37.0 \mathrm{~mW}, 0 \mathrm{~mW})$ and $(0 \mathrm{~mW}, 41.5 \mathrm{~mW})$ with frequencies 2.29 and $2.35 \mathrm{~Hz}$, respectively.

Figure 2(a) shows the reverse of a PS transition with increasing the input power. Up to $P_{e}=101.5 \mathrm{~mW}$ both oscillators show single-frequency periodic signals of different amplitudes but with the same frequency and a constant phase difference $\theta$ that changes with $P_{e}$. For $P_{e}$ values above $101.5 \mathrm{~mW}$, the system exhibits irregularly separated phase jumps in which one oscillator makes one more oscillation than the other in a short time interval. With increasing $P_{e}$, the jumps happen more frequently and become regularly separated, while the mean slope of $\theta(t)$ increases. The phase jumps manifest the tendency of the units to desynchronize and to differentiate their mean oscillation frequencies. The jump process is pointed out in more detail in Fig. 2(b) where the oscillatory signals of both units for $103 \mathrm{~mW}$ are shown together with the evolution of the time interval between successive oscillatory maxima. Notice that the periods of both units are continuously varying, that they become equal during the long intervals of constant $\theta$ and that the phase slips occur through strong variations of both periods during which one unit oscillates faster than the other. Similar variations also appear in the oscillation amplitudes. The Fourier spectra shown in Fig. 3 denote the appearance of a quasiperiodic state with the progressive differentiation of a second frequency and the gradual predominance of one or another frequency on each unit. During the transition the width of the initial frequency peak grows by developing a large number of equidistant sidebands with gradually increasing spacing, as determined by the frequency of phase jumps, and, finally,

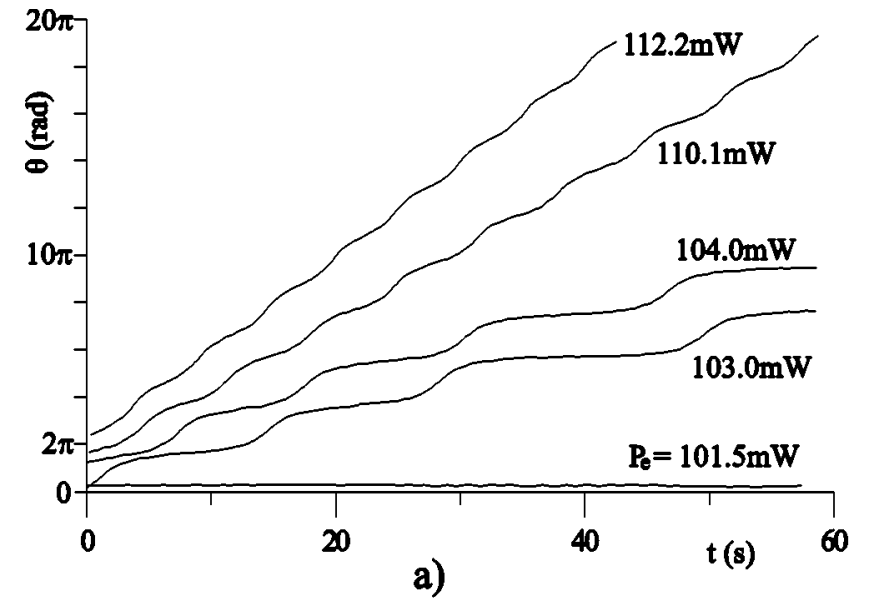

$103.0 \mathrm{~mW}$
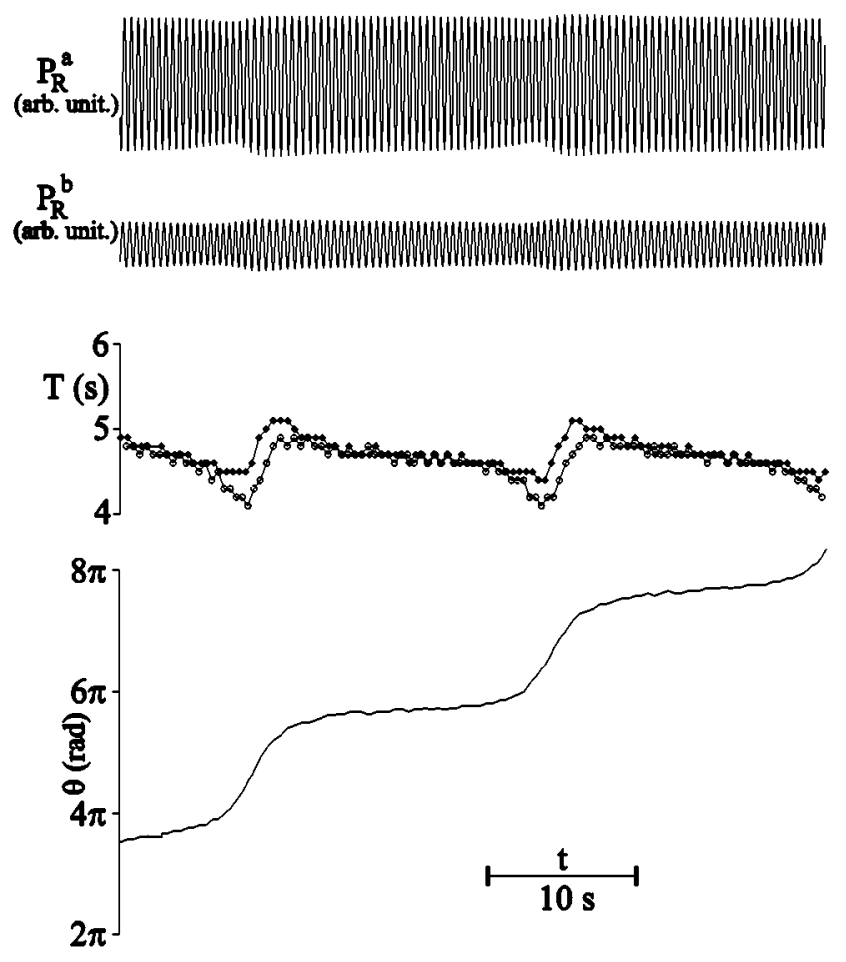

b)

FIG. 2. Experimental results illustrating a synchronization transition with phase jumps obtained by decreasing the total incident power in the coupled pair of two-layer oscillators. (a) Time evolution of the phase difference $\theta$ for different $P_{e}$ values. (b) Detail of a pair of phase slips for $P_{e}=103.0 \mathrm{~mW}$, as seen in the time evolutions of the output powers and of the time interval $T$ between succesive oscillatory maxima of both units.

the two units become dominated by contiguous sidebands while the number of peaks decreases.

Figure 4 presents numerical simulations for a configuration similar to the experimental case and with the parameter values given in the caption. These results show the same kind of transition to synchronization by phase jumps with very similar time evolutions and power spectra. In both experiment and simulation, $\theta(t)$ does not reach the straight 
Reflected power of a
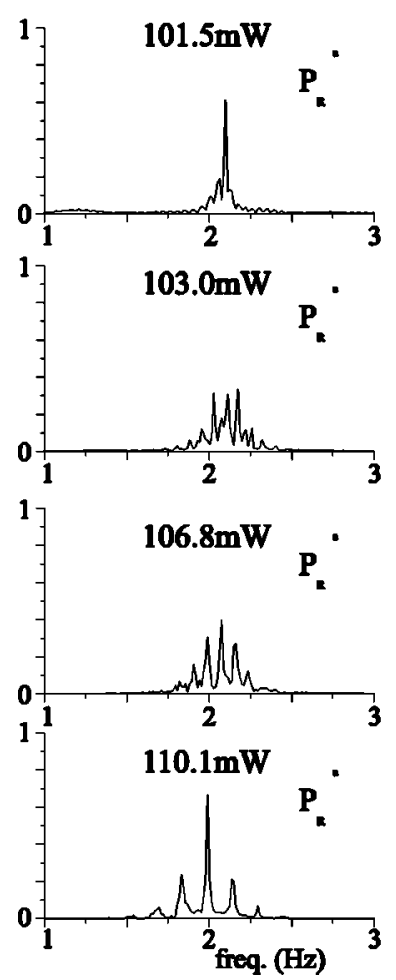

Reflected power of $\mathbf{a}$ and $b$
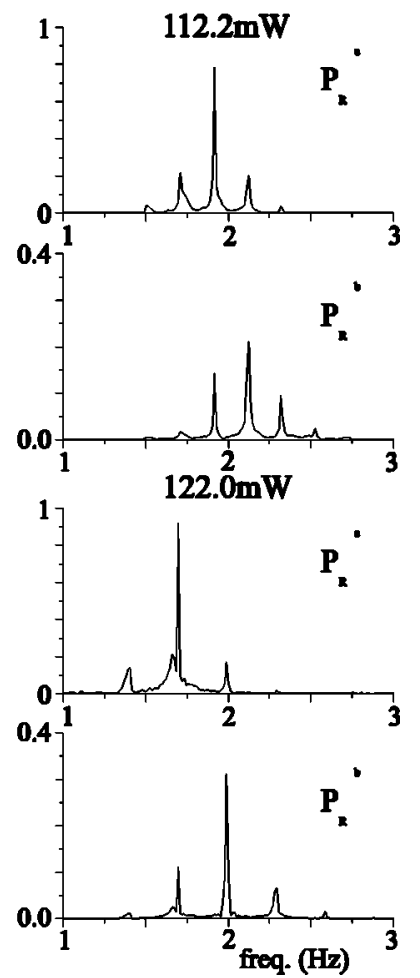

FIG. 3. Fourier spectra of the reflected powers $P_{R}^{a}$ and $P_{R}^{b}$ for different input powers, corresponding to the case of Fig. 2.

line of independent oscillations that would correspond to the absence of coupling because this is not possible by increasing the light power.

As is known from the periodically forced systems, the above transition between two- and single-frequency states may be associated with the penetration within the inner part of the period- 1 tongue by crossing two cyclic saddle-node bifurcations. The process is usually reversible and, beginning from the quasiperiodic state, it successively includes the periodic pulling with regular phase jumps, the noise enhanced type-I intermittencies, the locked state on a resonant torus

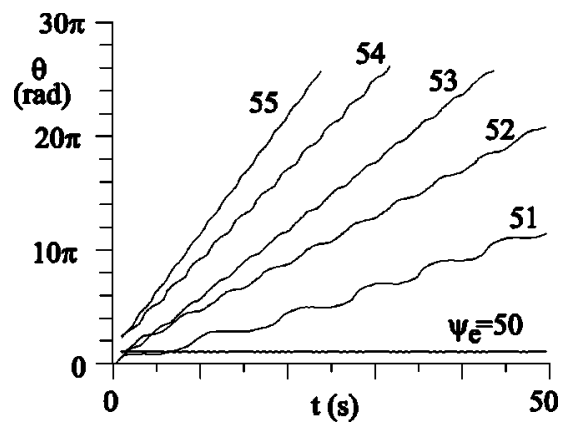

FIG. 4. Numerical simulation of the time evolution of the phase difference $\theta$ for different $P_{e}$ values. Following the dimensionless notation defined in Ref. [26], the parameters of the cavity spacer are $\eta_{1,2}=1,-10, g_{1,2}=1,0.5, K_{1,2}=D_{1,2}=1,0.1, h_{F}=h_{B}=0.5, \psi_{0}^{a}$ $=\pi, \psi_{0}^{b}=2$, the separating distance is $d=5.6$, and the normalized input powers are $\psi_{e}^{a}=\psi_{e}^{b}=\psi_{e} / 2$, with $\psi_{e}$ the total input power.

(after the first saddle-node bifurcation), and the free periodic orbit (after the second bifurcation where the saddle orbit vanishes with the unstable node orbit from which the invariant torus was created) that will finally shrink on the fixed point in the Hopf bifurcation [29]. Hysteretic transitions involving additional global bifurcations can also occur [8] but their experimental characterization is rather difficult.

\section{B. Transition through a secondary Hopf bifurcation}

It is also known from the periodically forced systems that the transition between single- and two-frequency states can occur directly through a torus or secondary Hopf bifurcation and, although accompanied by complex features, such a kind of transition was observed in our bidirectionally coupled oscillators by slightly decreasing the oil layer to $100 \mu \mathrm{m}$ and the distance $d$ to $5.35 \mathrm{~mm}$. In the experimental results of Fig. 5 it may be seen that the periodic orbit with constant $\theta$ (35.1 $\mathrm{mW}$ ) born in a Hopf bifurcation of the stationary solution, experiences a torus bifurcation at $35.3 \mathrm{~mW}$, as denoted by the appearance of a low-frequency modulation with gradually increasing depth in the oscillatory signals and of a second frequency in the power spectrum of unit $a$. The new frequency does not become noticeable in the unit $b$ spectrum up to higher light powers. For a narrow $P_{e}$ interval the oscillators evolve with equal mean frequency but with alternate phase slips yielding low-amplitude oscillations in $\theta$ at the frequency difference of the two Fourier components (35.5 $\mathrm{mW})$. At a certain power this almost-synchronized $\theta$-oscillating state switches suddenly to another quasiperiodic state in which the two units have different mean frequencies due to the dominance of one or another Fourier component and $\theta(t)$ grows with the corresponding mean slope while the regular phase slips remain $(35.9 \mathrm{~mW})$. The comparison of these spectra with those of Fig. 3 clearly shows the different way through which the second frequency appears in the two kinds of transitions between periodic and quasiperiodic evolutions. The torus bifurcation creates a second frequency distinct from the first one, while the intermittent locking differentiates the second frequency from the first one by gradually increasing the frequency of jumps.

The numerical results presented in Fig. 6 show a similar but richer process when the normalized input power $\psi_{e}$ is increased: the fixed point makes a subcritical Hopf bifurcation followed by successive cyclic saddle-node bifurcations creating periodic orbits with $\theta$ alternatively next to 0 or $\pi$ [Fig. 6(a)]. The coexistence of orbits with different $\theta$ occurs in very narrow intervals of the input power and this might explain their absence in the experimental observations. Such a multiplicity of periodic orbits and their $\theta$ preferences have been analitically predicted with a simple phase model for a coupled pair of oscillators [30]. For larger $\psi_{e}$ values, the periodic orbit (denoted $\alpha$ in Fig. 6) becomes unstable through a subcritical secondary Hopf bifurcation giving rise to a quasiperiodic solution [denoted $\beta$ in Fig. 6(b)] that shows $\theta$ oscillations very similar to those of the experimental signal for $35.5 \mathrm{~mW}$. As in the experiment, this state vanishes at a certain $\psi_{e}$ value and the system switches to another quasiperiodic state with two different mean frequencies (de- 

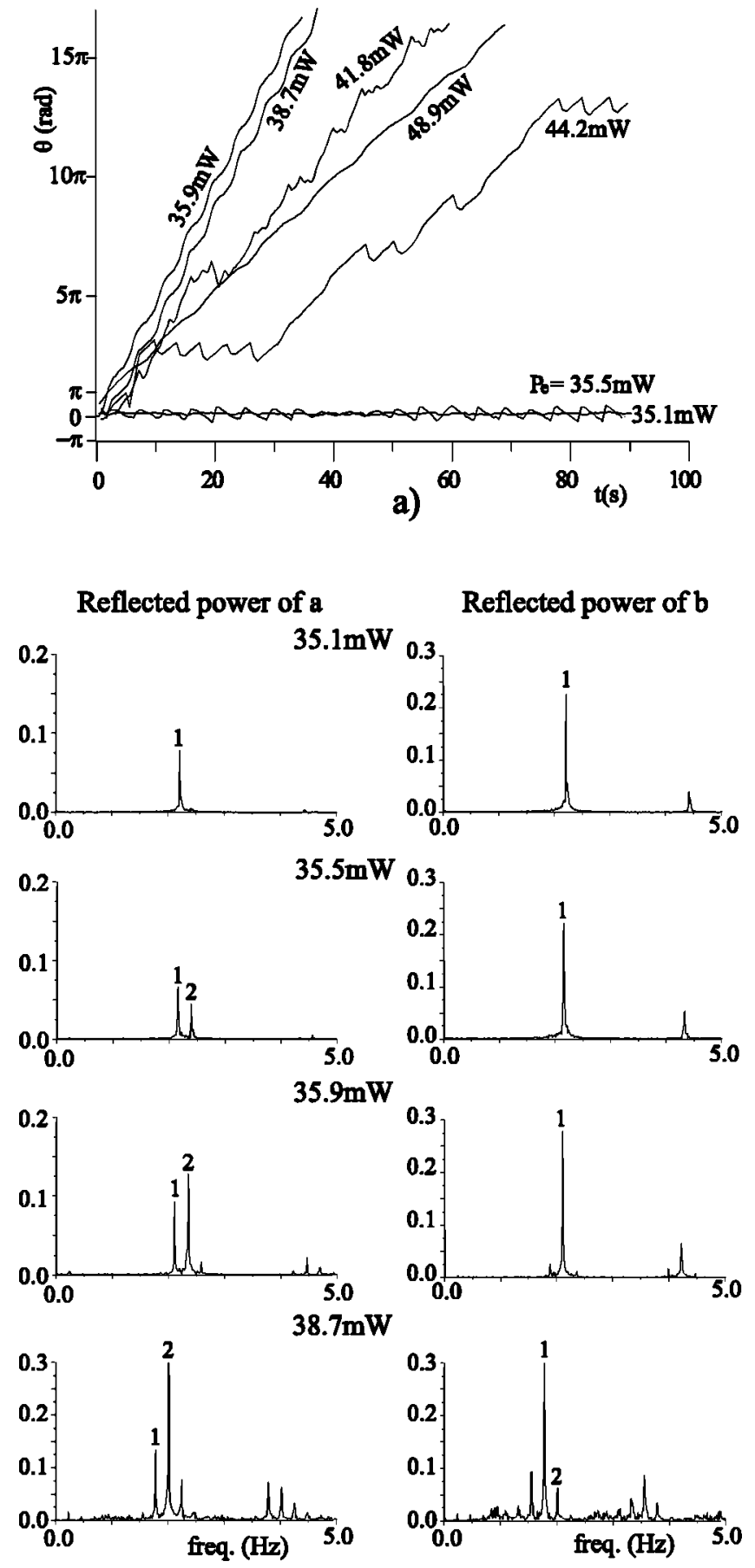

b)

FIG. 5. Synchonization transition associated with a torus bifurcation observed in a pair of two-layer oscillators slightly more disimilar than in the case of Fig. 2. (a) Time evolution of the phase difference $\theta$ for different $P_{e}$ values. (b) Fourier spectra of the reflected signals $P_{R}^{a}$ and $P_{R}^{b}$ for different input power values. Labels 1 and 2 relate peaks of equal frequency in the spectra of the two units.

noted $\delta$ ), while a third quasiperiodic state $\chi$ coexists also for a certain $\psi_{e}$ interval. It is reasonable to suspect saddle-node connections between such quasiperiodic solutions. The multiplicity of solutions seems to be intrinsic for mutually coupled nonlinear systems because the nonlinear part of the
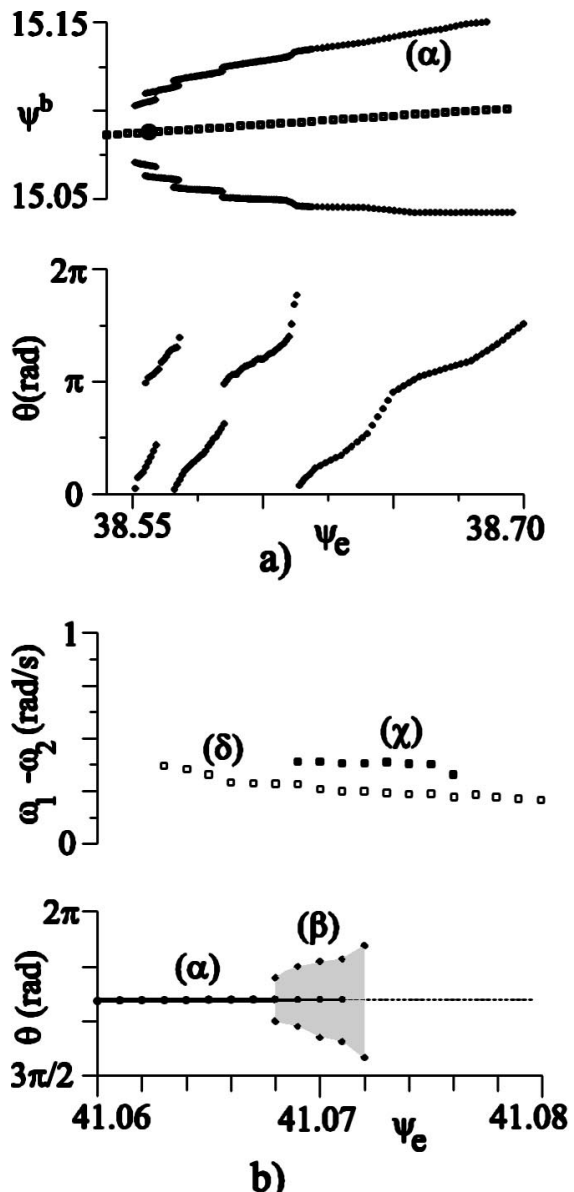

FIG. 6. Numerical simulation for the same parameters as in Fig. 4 but $d=8.0$ pointing out a complex sequence of bifurcations in a synchronization transition associated with the torus bifurcation. (a) Bifurcation diagram representing the total phase shift of device $b$ (in the coupled system) and the relative phase $\theta$ as a function of $\psi_{e}$ near the Hopf bifurcation of the fixed point, which occurs subcritically at $\psi_{e}=38.57$. The periodic state $\alpha$ derives from a sequence of cyclic saddle-node bifurcations. (b) Continuation of the bifurcation diagram for higher $\psi_{e}$ values by representing either the relative phase $\theta$, for states with almost constant $\theta$ values, or the mean frequency difference between the two units for the quasiperiodic solutions with increasing $\theta$.

associated vector field includes two linearly independent components and this means possibilities for a higher degree of multiplicity in both the stationary and oscillatory solutions.

The experimental results of Fig. 5 also show how the phases and amplitudes of the two coupled oscillators may be differently correlated. Increasing $P_{e}$ from $35.9 \mathrm{~mW}$ to 38.7 $\mathrm{mW}$, the whole system evolution changes from quasiperiodic to chaotic, the amplitudes become largely uncorrelated but $\theta(t)$ remains almost unchanged. The lack of amplitude correlation is pointed out by the reflected power time evolutions [Fig. 7(a)] and more clearly by the Poincaré sections of the reconstructed attractors. The recontructions in a plane of section exclusive of subsystem $b$ [Fig. 7(b)] show patterns corresponding to quasiperiodic and chaotic signals for $35.9 \mathrm{~mW}$ and $38.7 \mathrm{~mW}$, respectively. The same attractors reconstructed 

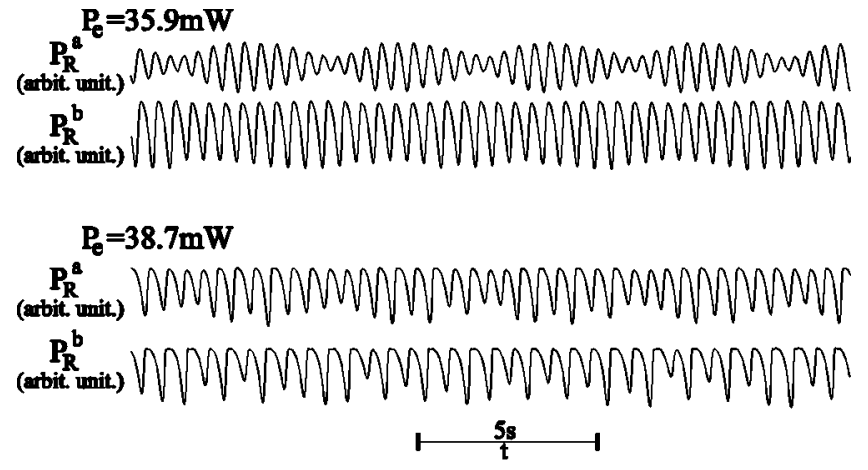

a)

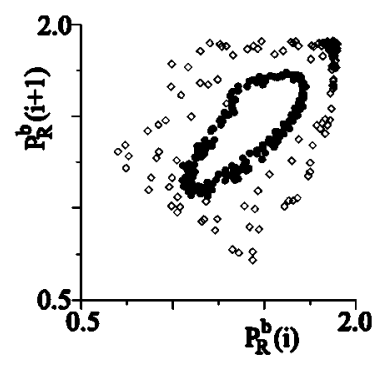

b)

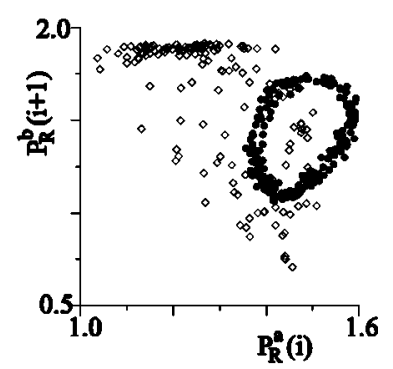

c)
FIG. 7. (a) $P_{R}^{a}$ and $P_{R}^{b}$ time evolutions for $P_{e}=35.9 \mathrm{~mW}$ and $38.7 \mathrm{~mW}$. (b) Poincare sections of embedded attractors from the $P_{R}^{b}$ time evolution for $P_{e}=35.9 \mathrm{~mW}(\bullet)$ and $38.7 \mathrm{~mW}(\diamond)$. (c) Poincare sections in the $\left(P_{R}^{a}, P_{R}^{b}\right)$ plane for the same cases as in (b) to point out the correlation degree between oscillators.

in a hybrid plane of the two subsystems [Fig. 7(c)] indicate a high correlation of amplitudes for $35.9 \mathrm{~mW}$ but a poor one for $38.7 \mathrm{~mW}$. It is an example of transition to chaos in the coupled pair without changes in the phase relationship between the two oscillators, i.e., both units evolve with the same mean frequencies and with the same regular phase slips as before, while the amplitudes has lost correlation. With further increasing $P_{e}$ the system evolution remains chaotic while $\theta(t)$ becomes first irregular $(41.8 \mathrm{~mW})$ and then tends to restabilization by showing intervals of $\theta$ oscillations conected by $2 \pi$ jumps $(44.2 \mathrm{~mW})$. This $\theta$ behavior has been also found in simulations of sets of coupled phase oscillators near phase synchronization transitions [31]. For $P_{e}$ values near $50 \mathrm{~mW}$ the system recovers quasiperiodicity, although with a lower mean frequency difference.

\section{TWO COUPLED THREE-DIMENSIONAL SUBSYSTEMS}

Phase synchronization transitions have been also observed in coupled oscillators created on a three-layer BOITAL device [32], which already present chaotic evolutions when isolated. The whole system is described with a reduced model, equivalent to that used for the two-layer case, but only considering heat transfer within the first layer of the device. The model is written as

$$
\begin{aligned}
\frac{d \psi_{1}^{a}}{d t}= & -b_{11} \psi_{1}^{a}-b_{12} \psi_{2}^{a}-b_{13} \psi_{3}^{a}+G_{1} A\left(\psi^{a}\right) \psi_{e}^{a} \\
& +c_{1} \psi_{1}^{b}\left(t-\tau_{1}\right),
\end{aligned}
$$
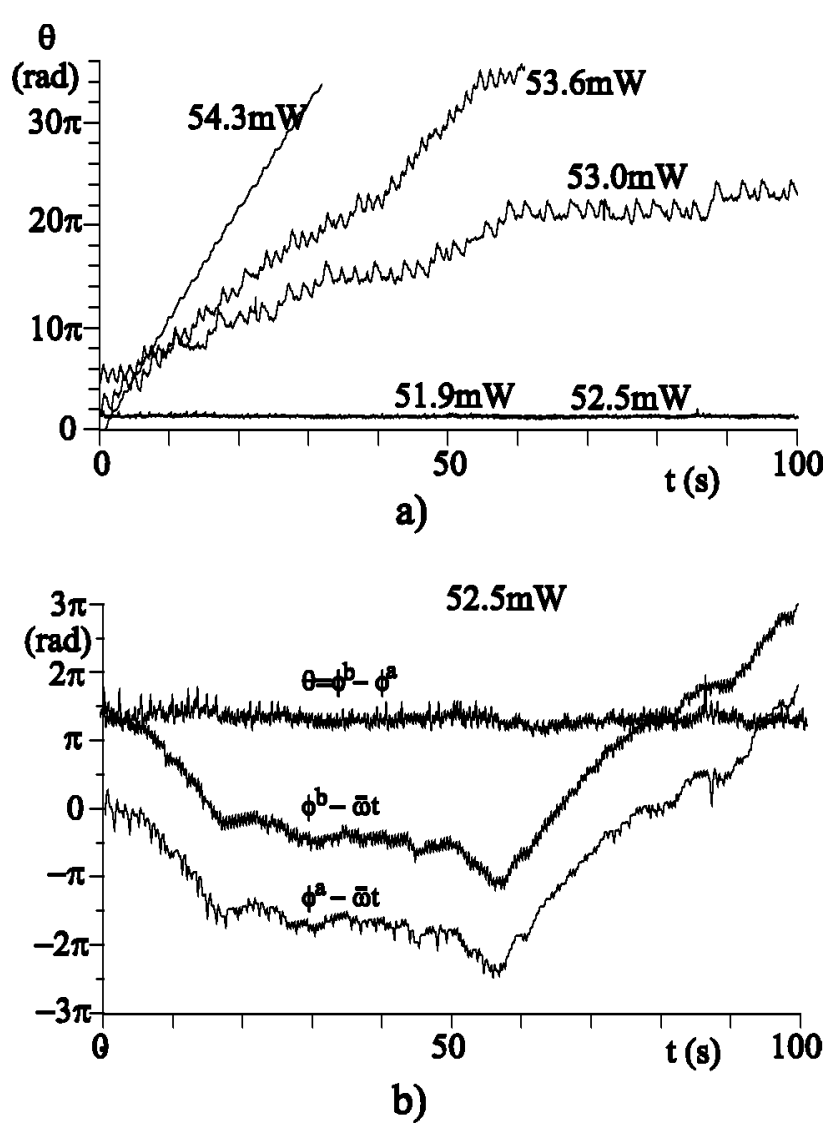

FIG. 8. Experimental results illustrating a synchronization transition observed in a coupled pair of three-layer BOITAL oscillators. (a) Time evolution of $\theta$ for different input light powers. (b) Vertically enlarged representation of $\theta(t)$ for $P_{e}=52.5 \mathrm{~mW}$ showing the occurrence of fast oscillations. The time variation of the individual phases of the coupled oscillators with respect to the harmonic oscillation at the mean frequency $\bar{\omega}$ is also represented.

$$
\begin{aligned}
& \frac{d \psi_{2}^{a}}{d t}=-b_{21} \psi_{1}^{a}-b_{22} \psi_{2}^{a}-b_{23} \psi_{3}^{a}+G_{2} A\left(\psi^{a}\right) \psi_{e}^{a}, \\
& \frac{d \psi_{3}^{a}}{d t}=-b_{31} \psi_{1}^{a}-b_{32} \psi_{2}^{a}-b_{33} \psi_{3}^{a}+G_{3} A\left(\psi^{a}\right) \psi_{e}^{a},
\end{aligned}
$$

for subsystem $a$ and the same equations but exchanging $a$ with $b$ for the other subsystem. The coefficients $b_{i j}$ and $G_{i}$ depend on parameters of the spacing layers and the nonlinear function $A\left(\psi^{x}\right)$ depends on the mirror properties [26]. The simplification of the coupling scheme by disregarding heat coupling towards the second and third layers makes the integration easier and we expect that this has not relevant consequences because equivalent results are obtained with one or two time delays in the two-layer case.

The results of Figs. 8-13 correspond to a pair of oscillators generated in a BOITAL device made of $150 \mu \mathrm{m}$ of glass, $35 \mu \mathrm{m}$ of sunflower oil and $1 \mathrm{~mm}$ of glass, with equal input powers but different initial phase shifts, and a separation of $d=6 \mathrm{~mm}$. Figure 8 presents the time evolution of $\theta$ for different incident powers and Fig. 9 shows the time evolutions of both oscillators for the three lower powers. After 


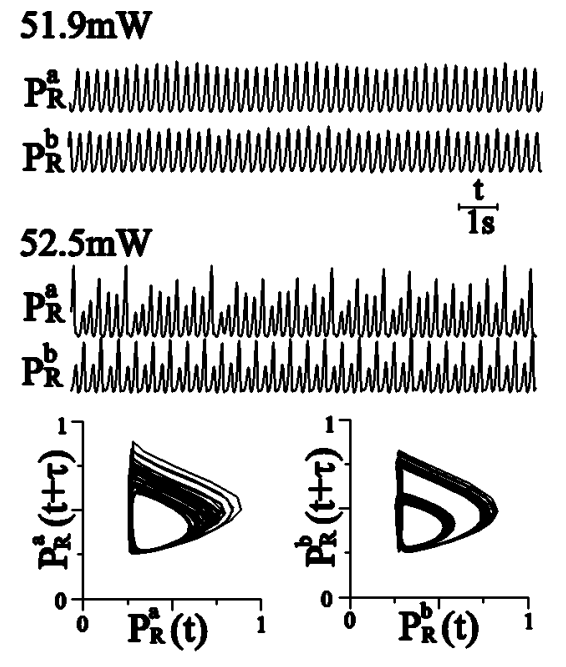

\section{$53.0 \mathrm{~mW}$}
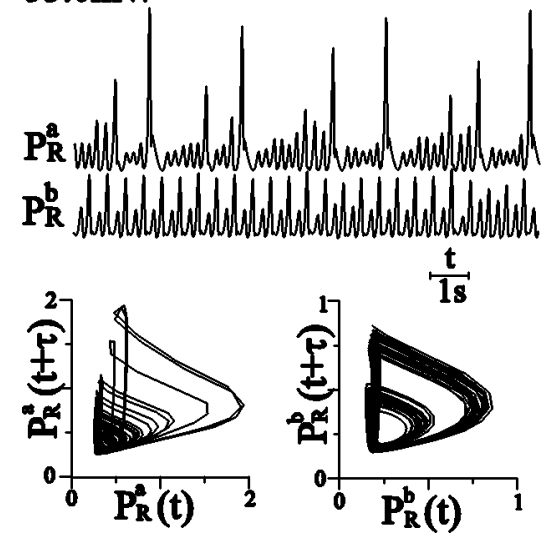

FIG. 9. Time evolution of the reflected powers $P_{R}^{a}$ and $P_{R}^{b}$ and embedded attractors for the three lower $P_{e}$ values of Fig. 8.

the Hopf bifurcation of the stationary solution, the system evolves with a single-frequency periodic oscillation that, for $P_{e}=51.9 \mathrm{~m}$, begins to exhibit slight amplitude irregularities although the phase difference remains constant about $4 \pi / 3$ $\pm \pi / 10$. For $P_{e}=52.5 \mathrm{~mW}$ the system has experienced a period doubling unequally affecting the two subsystems. It mainly affects oscillator $b$ and the two oscillators describe rather different wave form evolutions. The amplitudes and phases of both the subsystems vary irregularly but, while there is a lack of amplitude correlation, the phases tend to remain synchronized. The lack of correlation between the subsystem amplitudes is pointed out by the section of the embedded attractor represented in Fig. 10, while the synchronization of phases is visible in Fig. 8(b). The phase of each oscillator presents large drifts but $\theta(t)$ remains confined within an interval lower than $\pi$ by describing oscillations with a frequency equal to the half of the oscillatory frequency. Such $\theta$ oscillations are clearly associated with the period doubling that mainly affects oscillator $b$.

The differentiation of the units enhances when $P_{e}$ is slightly increased. The $\theta(t)$ fluctuations become of lower frequency but of amplitude as large as about $2 \pi$ and from time to time a $2 \pi$ phase jump remains unrecovered $\left(P_{e}\right.$

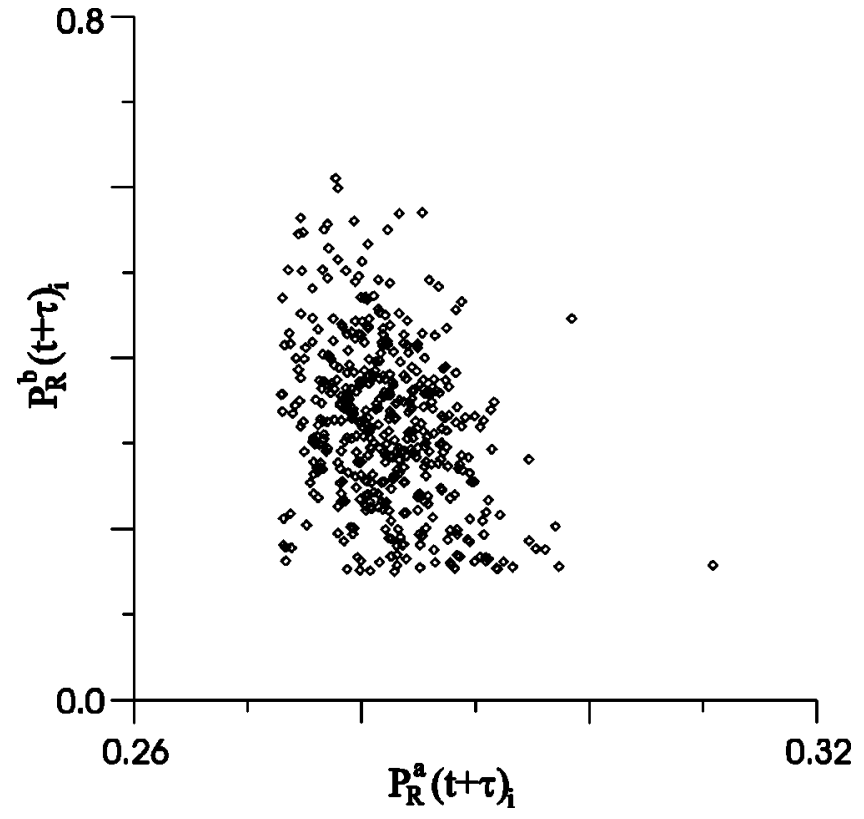

FIG. 10. Section of the reconstructed attractor for $P_{e}$ $=52.5 \mathrm{~mW}$ pointing out the lack of correlation between the two reflected powers. The attractor has been embedded in $\left[P_{R}^{a}(t)\right.$, $\left.P_{R}^{a}(t+\tau), P_{R}^{b}(t), P_{R}^{b}(t+\tau)\right]$, with $\tau$ a fourth of the oscillation period, and the section is for a constant value of $P_{R}^{a}(t)$.

$=53.0$ and $53.6 \mathrm{~mW}$ ). The number of unrecovered phase jumps increase with the input power and at a certain power become rather regular $\left(P_{e}=54.3 \mathrm{~mW}\right)$. The spectra presented in Fig. 11 illustrate how at the end of the transition the two units oscillate with different frequencies, i.e., the Fourier components of one unit are almost absent in the other, but it is not clear how the new frequency appears during the transition. The subharmonic peak in the spectra of both units points out the period doubling process occurring below 52.5 $\mathrm{mW}$. The initial frequency components remain always dominant in subsystem $b$ without noticeable influence of additional frequencies, while in subsystem $a$ the subharmonic of the initial frequency becomes stronger than the fundamental peak and a broad spectrum emerges with several relevant peaks. Finally, a qualitative change happens in between 54 and $54.3 \mathrm{~mW}$ in which the subharmonic structures of both the units dissapear and the system ends on a two-frequency chaotic state with a clear dominance of one of the frequencies on each subsystem and with a well regular $\theta(t)$ evolution. The final frequencies of subsystems $b$ and $a$ seem to be related to the initial frequency and its subharmonic, respectively. Comparison with the typical behavior of a periodically-forced oscillator suggests that the process might be associated with the saddle-node bifurcation of the period-2 tongue, within which period-doubling bifurcations are intrinsically involved [29]. Nevertheless, the lack of a fixed frequency in the present case of bidirectionally coupled oscillators makes rather difficult to characterize the process.

Figure 12 shows the evolution of the Lyapunov exponents with the incident light power through the PS transition, as well as the change of the difference between the mean frequencies of the two oscillators. The exponents have been 


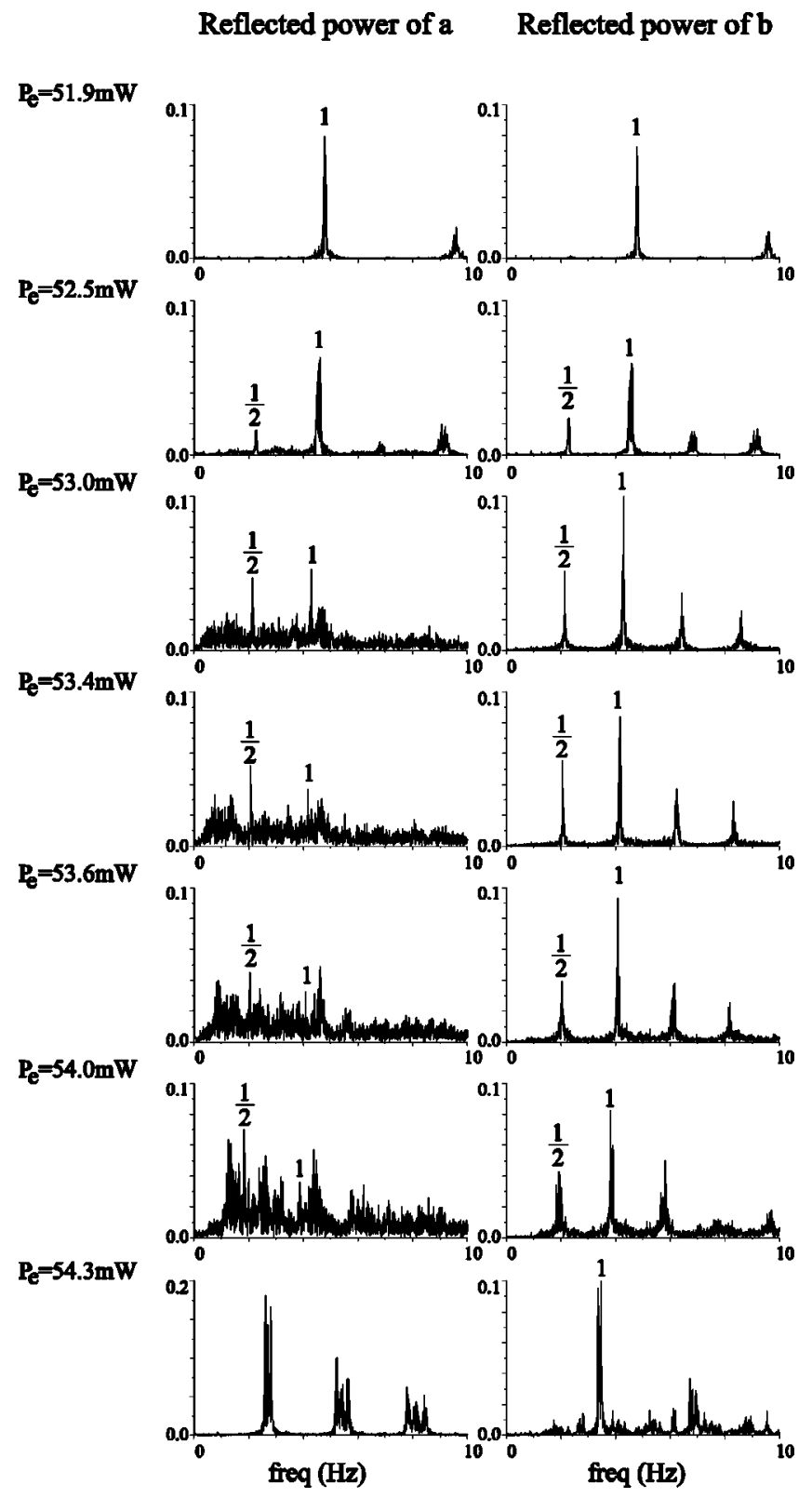

FIG. 11. Fourier spectra of both reflected light signals for different input powers, corresponding to the transition of Fig. 8. The labels 1 and 1/2 denote the peaks of the initial frequency and its subharmonic.

determined from a six-dimensional embedding based on the reflection outputs of the two oscillators. Notice the presence of two positive, one vanishing, and three negative exponents, one of which manifests a tendency to vanish just when the frequency difference increases markedly and the phase synchronization is lost. This tendency would be in agreement with the behavior found in the Rössler model by Rosenblum et al. [3], in which one of the two vanishing exponents existing in the absence of coupling becomes clearly negative during the PS transition as a consequence of the interdependence between the phases of the two oscillators.

The details of a synchronization transition may vary significantly by slightly changing the parameters of the device and this occurs associated with changes in the actual bifur-
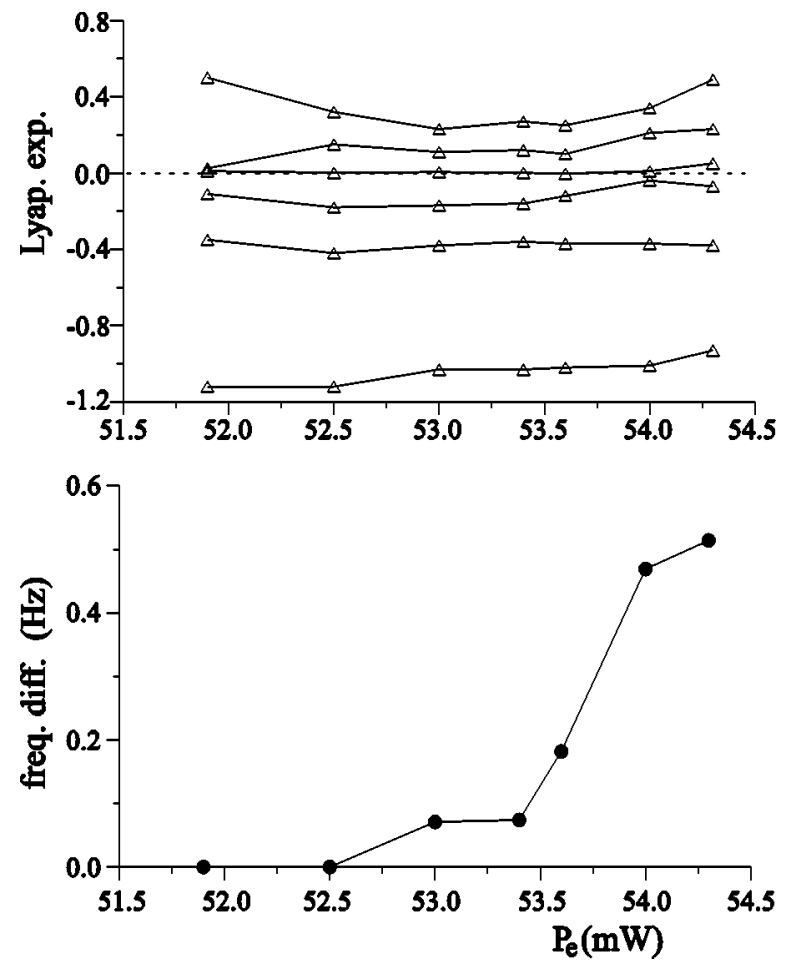

FIG. 12. Lyapunov exponents and frequency difference evolution when the input power is increased. A tendency of one of the negative Lyapunov exponents to vanish can be observed between 53.5 and $54.0 \mathrm{~mW}$.

cational sequence experienced by the system. For instance, Fig. 13 illustrates a PS transition observed with the same device as in Fig. 8 and for the same conditions, except for a slightly different value of the background temperature regulated by the thermoelectric plate. This temperature modifies the initial phase shift $\psi_{0}^{x}$ of the cavity roundtrip for the two nonlinear oscillators and consequently affects the nonlinear functions of both subsystems. In the case of Fig. 13, the synchronized state does not exhibit the period-doubling bifurcation, and the phase jumps of the intermediate states appears with a clear step-by-step succession and flat plateaus. A similar contrast of behaviors may be seen in the numerical results of Fig. 14, where two PS transitions corresponding to the same parameter values except for the initial light phase shifts are represented. Figure 14(a) corresponds to an almost symmetric case with a rather small difference between the initial light phase shifts of the two subsystems. As in the experimental case of Fig. 8, the synchronized state begins the desynchronization transition by showing oscillations in the relative phase $\theta(t)$ at a frequency equal to half of the oscillating frequency, which are clearly associated with a perioddoubling bifurcation, and the intermediate states show plateaus with fluctuating $2 \pi$ jumps. Figure 14(b) corresponds to more different values of the initial phase shift for the subsystems and the desynchronization process is similar to the experimental case of Fig. 13. The long intervals of constant $\theta$ between successive phase jumps indicate a stronger tendency to sy nchronization, as compared to the case of Fig. 14(a), and it can be attributed to the dominance of one subsystem on the other due to the asymmetry introduced by the initial phase shifts. 

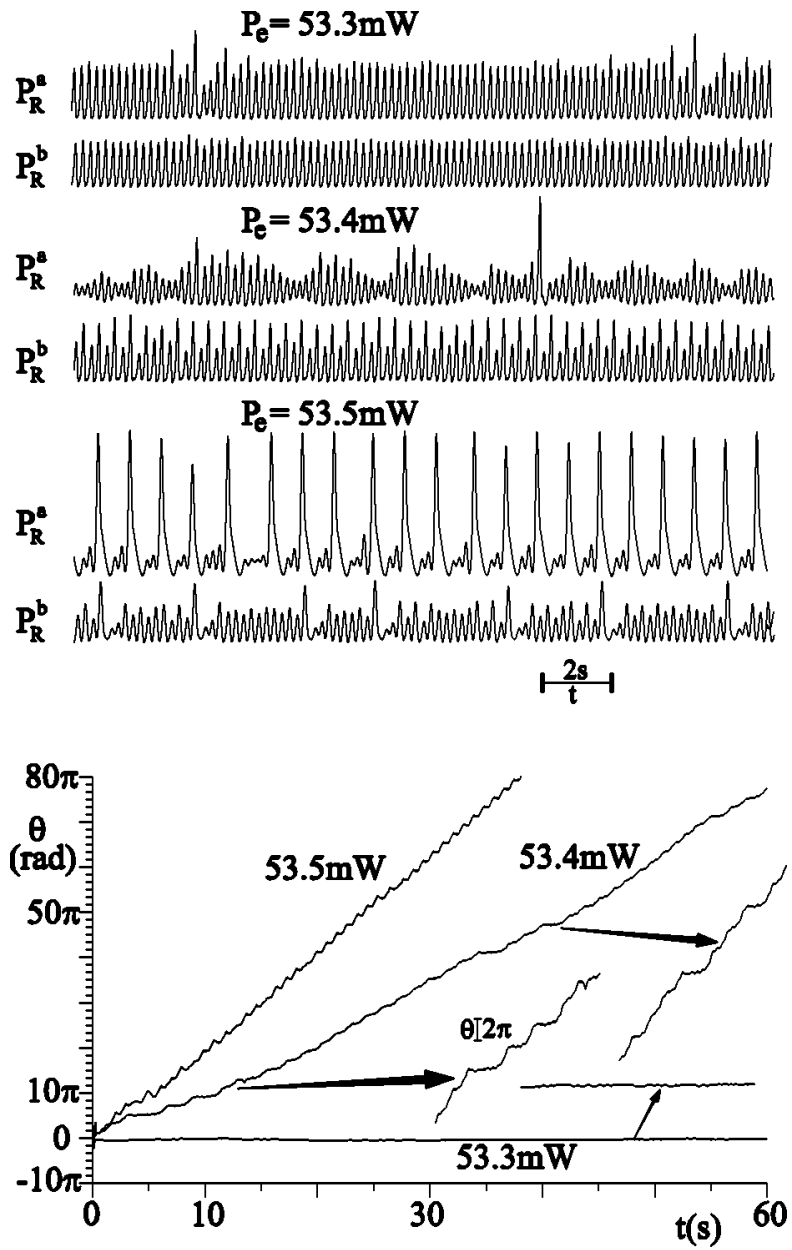

FIG. 13. Phase synchronization transition observed in the same experimental conditions as in Fig. 8 but with a background temperature of $20.6{ }^{\circ} \mathrm{C}$ instead of $20.3{ }^{\circ} \mathrm{C}$ : The differences between the two cases must be attributed to changes of the initial phase shifts of the interferometric cavity.

\section{CONCLUDING REMARKS}

In conclusion, we have investigated the relative behavior of bidirectionally coupled pairs of slightly different nonlinear oscillators as a function of the nonlinearity strength. The analysis has been done by using pairs of two-dimensional and three-dimensional oscillators in order for the individual subsystems to be able or not to exhibit chaos when isolated. A variety of desynchronization transitions from singlefrequency locked states to two-frequency states, where each subsystem tends to be dominated by its own frequency have been observed and characterized in detail. Intermediate states with the characteristic $2 \pi$ phase jumps generically appear during the transitions, specially in those clearly associated with a saddle-node bifurcation. Phase synchronization states with uncorrelated amplitudes have also been usually found, but for narrow ranges of the control parameter and always at the onset of chaos. We have observed PS states with both units evolving either at equal or at different mean frequencies.

In comparison with the periodically forced oscillators, the analysis of a pair of mutually coupled oscillators is much
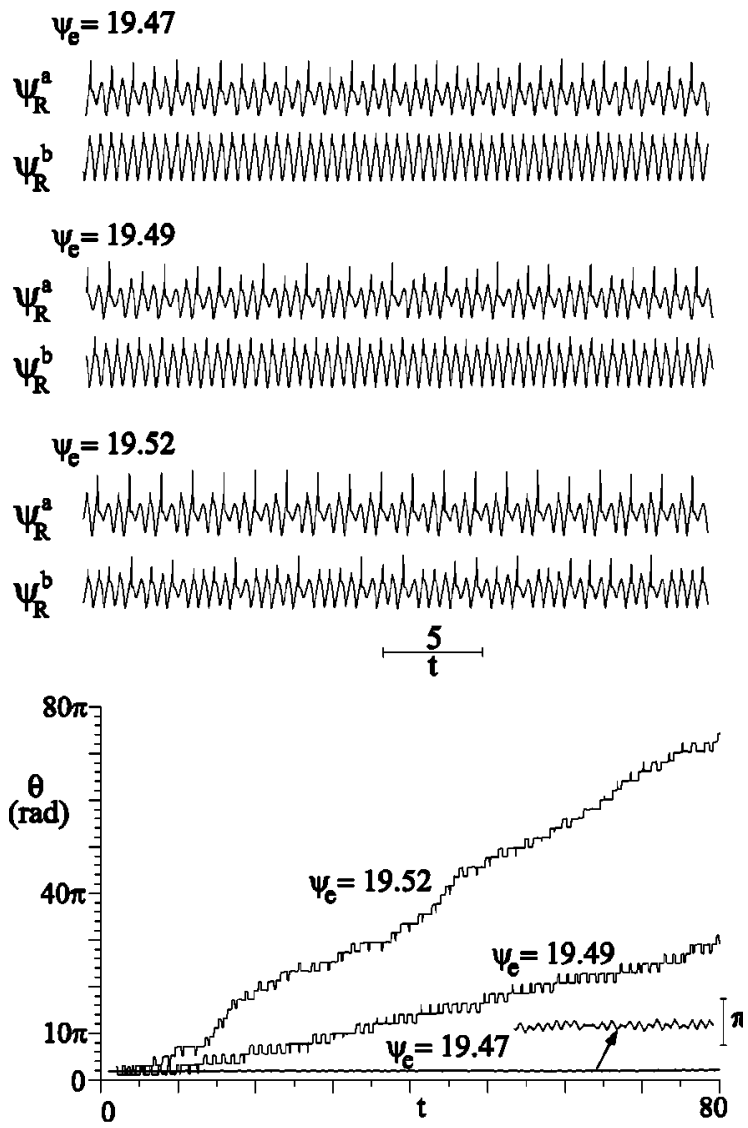

a)

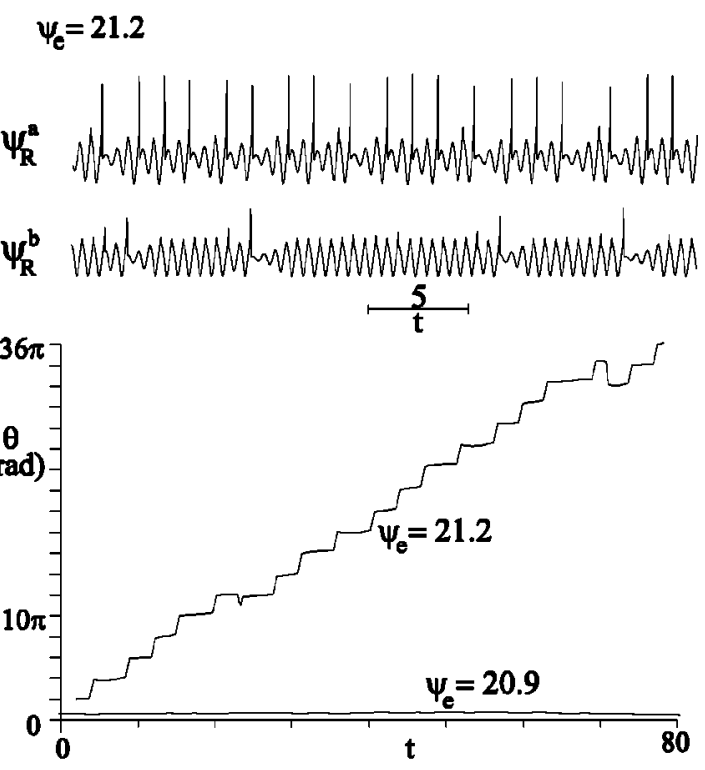

b)

FIG. 14. Numerical simulations illustrating the influence of a slight modification of the initial interferometric phase shifts on the PS transition. The dimensionless parameters [26] of the three-layer device are $\eta_{1,2,3}=1,-6,1 ; g_{1,2,3}=1,0.4,6 ; K_{1,2,3}=D_{1,2,3}=1$, $0.1,1$; and $h_{F}=h_{B}=5$; the separating distance is $d=5.6$; the input powers $\psi_{e}^{a}=\psi_{e}^{b}=\psi_{e} / 2$ and the initial interferometric phase shifts are (a) $\psi_{0}^{a}=0.01, \psi_{0}^{b}=0.03$ and (b) $\psi_{0}^{a}=0.0, \psi_{0}^{b}=0.3$. 
more difficult due to the lack of the fixed reference introduced by the pacing frequency. In the two-dimensional case, the PS transitions associated with either a saddle-node or a torus bifurcation have been clearly observed. The simplest situation corresponds to a saddle-node bifurcation of the period-1 locked state, in which the system response remains regular during the transition except for a range of intermediate states with intermittent phase jumps. In the case of a torus bifurcation, the presence of the second frequency promotes the desynchronization process by means of a state with both units at the same mean frequency but with oscillatory slips in $\theta$, and which quickly becomes a regular quasiperiodic state with different mean frequencies for the subsystems. The quasiperiodic regularity is then lost and chaos appears through a PS state with uncorrelated amplitudes followed by a sequence of complex phenomena.

In the case of three-dimensional oscillators, the desynchronization transitions appear with a so rich variety of features that make rather difficult the association of the process with a particular bifurcation of the whole system. The PS states with uncorrelated amplitudes have been found with both oscillators evolving either at equal or different mean frequencies and intermediate states with $2 \pi$ phase jumps always appear. The analysis of Lyapunov exponents indicates that the transition from states with both units at equal mean frequency to states with the units at different mean frequencies is accompanied by a tendency of a negative exponent to vanish.

The experimental observation of phase synchronization effects could be also investigated by introducing optical instead of thermal coupling between the optothermal oscillators. The main advantage will be the good control of the coupling degree without the necessity of modifying the external input power to adjust the nonlinearity strength. In addition, the reflected light will connect the oscillators without any frequency filtering, while heat diffusion damps with the frequency and the separation distance. Moreover, the optical coupling will be nonlinear and will introduce additional possibilities for complex behaviors.

\section{ACKNOWLEDGMENT}

Financial support from the Spanish DGES under Grant No. PB98-0899 is acknowledged.
[1] L.M. Pecora, T.L. Carroll, Phys. Rev. Lett. 64, 821 (1990).

[2] For a review, see A.S. Pikovsky, M.G. Rosenblum, and J. Kurths, Int. J. Bifurcation Chaos Appl. Sci. Eng. 10, 2289 (2000), and references therein.

[3] M. Rosenblum, A. Pikovsky, J. Kurths, Phys. Rev. Lett. 76, 1804 (1996).

[4] K. Lee, Y. Kwak, and T. Kun Lim, Phys. Rev. Lett. 81, 321 (1998).

[5] A. Pikovsky, G. Osipov, M. Rosenblum, M. Zaks, and J. Kurths, Phys. Rev. Lett. 79, 47 (1997).

[6] E. Rosa, E. Ott, and M.H. Hess, Phys. Rev. Lett. 80, 1642 (1998)

[7] Z. Zheng, G. Hu, and B. Hu, Phys. Rev. Lett. 24, 5318 (1998).

[8] D.E. Postnov, A.G. Balanov, N.B. Janson, and E. Mosekilde, Phys. Rev. Lett. 83, 1942 (1999).

[9] I. Kim, C-M. Kim, W-H. Kye, and Y-J. Park, Phys. Rev. E 62, 8826 (2000).

[10] G.V. Osipov, A.S. Pikovsky, and J. Kurths, Phys. Rev. Lett. 88, 054102 (2002).

[11] E-Y. Park, M.A. Zaks, and J. Kurths, Phys. Rev. E 60, 6627 (1999).

[12] W-H. Kye and C-M. Kim, Phys. Rev. E 62, 6304 (2000).

[13] A.S. Pikovsky, Sov. J. Commun. Technol. Electron. 30, 85 (1985).

[14] C.M. Ticos, E. Rosa, W.B. Pardo, J.A. Walkenstein, and M. Monti, Phys. Rev. Lett. 85, 2929 (2000).

[15] E. Allaria, F.T. Arecchi, A. Di Garbo, and R. Meucci, Phys. Rev. Lett. 86, 791 (2001).

[16] I.Z. Kiss and J.L. Hudson, Phys. Rev. E 64, 046215 (2001).

[17] G.D. Funk, I.J. Valenzuela, and W.K. Milsom, J. Exp. Biol. 200, 915 (1997).
[18] A. Neiman, X. Pei, D. Russell, W. Wojtenek, L. Wilkens, F. Moss, H.A. Braun, M.T. Huber, and K. Voigt, Phys. Rev. Lett. 82, 660 (1999).

[19] U. Parlitz, L. Junge, W. Lauterborn, and L. Kocarev, Phys. Rev. E 54, 2115 (1996).

[20] D.Y. Tang, R. Dykstra, M.W. Hamilton, and N.R. Heckenberg, Phys. Rev. E 57, 3649 (1998).

[21] P. Tass, M.G. Rosenblum, J. Weule, J. Kurths, A. Pikovsky, J. Volkmann, and A. Schnitzler, H.-J. Freud, Phys. Rev. Lett. 81, 3291 (1998).

[22] J.P. Lachaux, E. Rodriguez, J. Martinerie, and F. Varela, Hum. Brain Mapp 8, 194 (1999).

[23] F. Varela, J.P. Lachaux, E. Rodriguez, and J. Martinerie, Nature Rev. Neurosci. 2, 229 (2001).

[24] K.V. Volodchenko, V.N. Ivanov, S-H. Gong, M. Choi, Y-J. Park, and C-M. Kim, Opt. Lett. 26, 1406 (2001).

[25] J.I. Rosell, J. Farjas, R. Herrero, F. Pi, and G. Orriols, Physica D 85, 509 (1995).

[26] J. Farjas, J.I. Rosell, R. Herrero, R. Pons, F. Pi, and G. Orriols, Physica D 95, 107 (1996).

[27] R. Herrero, R. Pons, J. Farjas, F. Pi, and G. Orriols, Phys. Rev. E 53, 5627 (1996).

[28] R. Herrero, M. Figueras, J. Rius, F. Pi, and G. Orriols, Phys. Rev. Lett. 84, 5312 (2000).

[29] For a review, see J. Farjas, R. Herrero, F. Pi, and G. Orriols, Int. J. Bifurcation Chaos Appl. Sci. Eng. 8, 1413 (1996).

[30] H. Schuster and P. Wagner, Prog. Theor. Phys. 81, 939 (1989).

[31] Z. Zheng, B. Hu, and G. Hu, Phys. Rev. E 62, 402 (2000).

[32] R. Herrero, F. Boixader, G. Orriols, J.I. Rosell, and F. Pi, Opt. Commun. 113, 324 (1994). 